\title{
Cavernous malformation of the optic pathway mimicking optic glioma: a case report
}

\author{
Yui Mano • Toshihiro Kumabe • Ryuta Saito • \\ Mika Watanabe • Teiji Tominaga
}

Received: 3 January 2014 / Accepted: 11 June 2014 / Published online: 3 July 2014

(C) Springer-Verlag Berlin Heidelberg 2014

\begin{abstract}
Purpose Optic pathway cavernous malformations (CMs) are extremely rare, accounting for less than $1 \%$ of all intracranial CMs. We report a case of optic pathway $\mathrm{CM}$ mimicking optic glioma because the initial magnetic resonance (MR) images did not disclose hemorrhagic findings such as popcorn-like lesion or hemosiderin ring.

Methods A 20-year-old woman presented with subacute left visual acuity loss and visual field defect and was referred to our hospital. Initial MR imaging findings were suggestive of optic glioma. Second MR imaging demonstrated hemorrhagic findings, but the hemorrhage was considered to be intratumoral hemorrhage from left optic pilocytic astrocytoma. She underwent radiochemotherapy and intravenous administration of corticosteroids but her symptoms deteriorated. Third and fourth MR imaging revealed enlargement of the hematoma. Therefore, the radiation therapy was interrupted at a delivered dose of $16.2 \mathrm{~Gy}$, and craniotomy was performed to preserve residual right visual field. The lesion was totally removed and the histological diagnosis was CM. Her right visual field was preserved, but not improved.

Results The present case and previous cases suggest that optic pathway CMs sometimes do not initially manifest with signs of hemorrhage, so CMs should be included in the differential diagnosis of optic pathway lesions.
\end{abstract}

\footnotetext{
Y. Mano · T. Kumabe $(\bowtie)$

Department of Neurosurgery, Kitasato University School of

Medicine, 1-15-1 Kitasato, Minami-ku, Sagamihara,

Kanagawa 252-0374, Japan

e-mail: kuma@kitasato-u.ac.jp
}

Y. Mano $\cdot$ R. Saito $\cdot$ T. Tominaga

Department of Neurosurgery, Tohoku University Graduate School of

Medicine, Sendai, Miyagi, Japan

M. Watanabe

Department of Pathology, Tohoku University Hospital, Sendai, Miyagi, Japan
Conclusions Histological confirmation with preparation for total resection should be considered for rapidly progressive cases even if the neuroimaging findings are compatible with optic glioma.

Keywords Optic cavernous malformation - Optic cavernous angioma $\cdot$ Optic cavernoma $\cdot$ Optic glioma $\cdot$ Differential diagnosis $\cdot$ Magnetic resonance imaging

\section{Introduction}

Cavernous malformations (CMs) are the second most common type of vascular lesion and account for $10-15 \%$ of all vascular malformations [2]. The incidence of $\mathrm{CMs}$ ranges from 0.4 to $0.6 \%$ of the population, with a male-to-female ratio of 1:1. More than half of cases of $\mathrm{CM}$ were located in the cerebral hemisphere, and multiple intracranial CMs were found in $19 \%$ of patients. The overall annual hemorrhage rate has been reported as $2.4 \%$ per patient-year (range from 1.6 to $3.1 \%$ ), and the rebleeding rate in several natural history studies varied widely from 4.5 to $22.9 \%$ per patient-year. The risk factors for bleeding were prior hemorrhage and female sex [8]. The true hemorrhage rates are quite controversial. Optic CM is a rare entity with an incidence of less than $1 \%$ of intracranial CMs. The major symptoms of optic CMs are visual deficit (98\%), including visual acuity loss, visual field defect, or both [11]. Optic gliomas represent approximately 3 $5 \%$ of childhood brain tumors and affect $11-30 \%$ of children with neurofibromatosis type 1 [3]. Histologically, optic gliomas are mostly pilocytic astrocytoma [6]. Optic gliomas and optic CMs are sometimes difficult to distinguish based on neuroimaging findings.

We describe a case of optic CM mimicking optic glioma due to the absence of hemorrhagic findings on the initial magnetic resonance (MR) images and show the detailed diachronic changes in the MR findings with the hemorrhagic and clinical symptoms. 

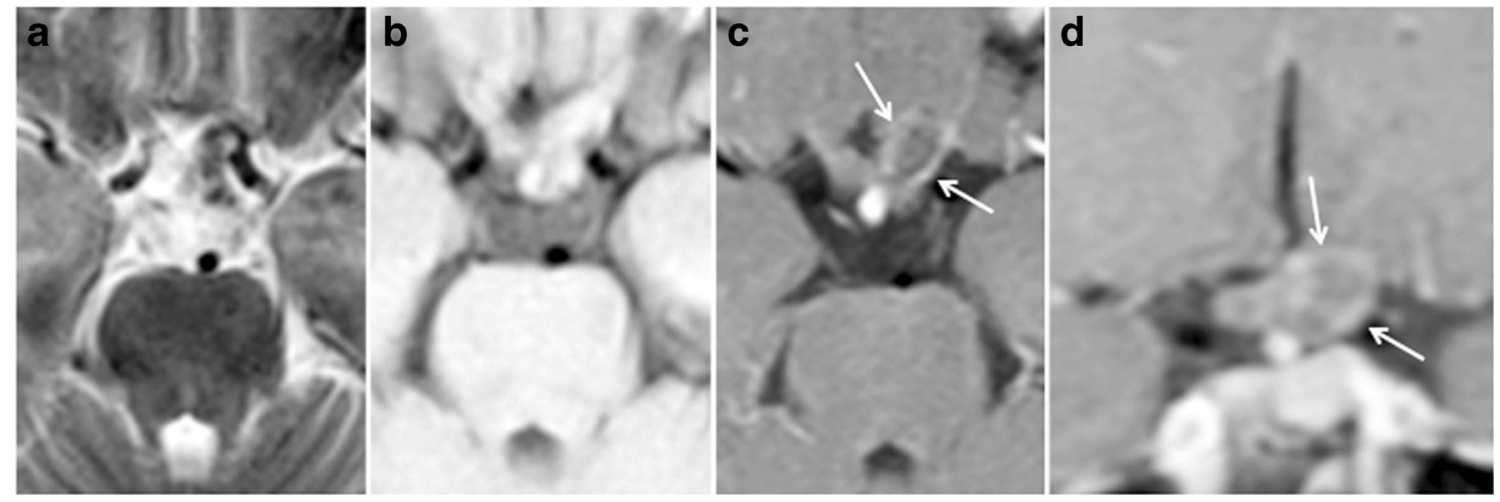

Fig. 1 Initial magnetic resonance (MR) images. Axial T2-weighted (a), and T1-weighted without (b) and with gadolinium (c) MR images; and coronal T1weighted MR image with gadolinium (d). First examination demonstrating swelling of the left optic nerve with peripheral rim-like enhancement (arrows)

\section{Case report}

A 20-year-old woman with no history of affective disorder including neurofibromatosis type 1 (NF1) suffered rapid progression of left visual acuity loss and visited another hospital. Initial MR imaging acquired at that hospital demonstrated swelling of the left optic nerve with peripheral enhancement (Fig. 1a-d). Left optic nerve glioma was suspected, and she was referred to our department 2 weeks after the initial MR imaging examination. Second MR imaging at our outpatient clinic demonstrated hemorrhagic change within the enhanced lesion, which was considered to be intratumoral hemorrhage from optic pilocytic astrocytoma (Fig. 2a-c). Carboplatin $450 \mathrm{mg} / \mathrm{m}^{2}$ was administered intravenously. Two weeks later, she had lost left visual acuity completely and had only right nasal visual field. Third MR imaging revealed enlargement of the hemorrhage (Fig. 3a-c), and she was immediately admitted to our department. She underwent corticosteroid administration and started to receive fractionated local radiotherapy to a planned dose of 45 Gy. MR imaging performed 2 weeks after the initiation of irradiation showed further enlargement of the hematoma (Fig. 4a-c). Therefore, the radiation therapy was discontinued after delivering a dose of $16.2 \mathrm{~Gy}$, and surgery was performed to preserve her right visual field. Left frontal osteoplastic craniotomy was performed, and a subfrontal approach was carried out. Old hematoma in various stages was evacuated, and a well-circumscribed mulberry-like lesion was excised from the left optic nerve and chiasm (Fig. 5a, b). The histological diagnosis was CM (Fig. 6a, b). No findings of malignancy or neuroglial tissues were observed among the sinusoid chambers. Postoperative MR imaging demonstrated total removal of the lesion (Fig. 7a-c). Her right visual field was preserved, but had not improved at 6 months after the surgery.

\section{Discussion}

Epidemiology and clinical course of optic pathway CMs

CMs of the optic pathway are extremely rare, with a review finding only 65 cases of optic pathway CM [11]. A further 3 cases have been reported since 2011 [12, 13, 17]. The most common presenting symptom was visual deficit (98\%), such as visual acuity loss, visual field defect, or both. Most patients $(60 \%)$ presented with headache or retro-orbital pain. The
Fig. 2 Second MR images, taken 2 weeks later just before the administration of carboplatin, disclosing the subacute phase of hemorrhage within the left optic nerve. Axial T2-weighted (a), and T1-weighted without (b) and with gadolinium (c) MR images. Figs. 2, 3, and 4 show the same arrangement of MR sequences
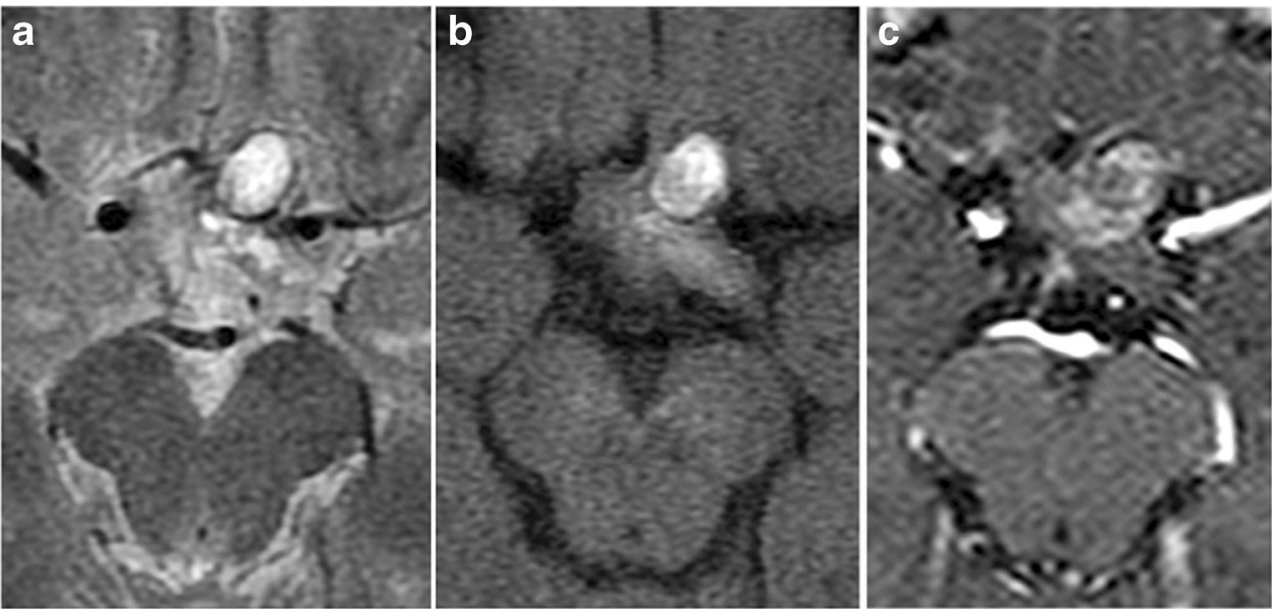
Fig. 3 Third MR images, after another 2 weeks just before the radiation therapy, depicting enlargement of the hemorrhage. The hemosiderin rim was apparent but not considered optic $\mathrm{CM}$ at that point
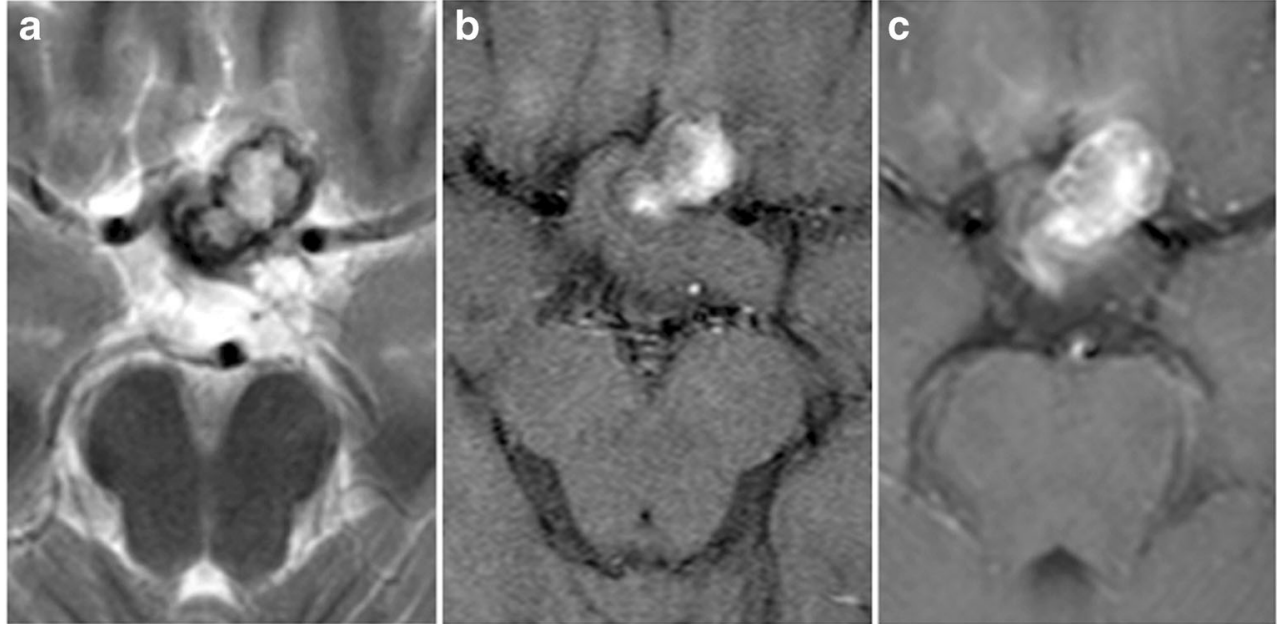

onset of symptoms was acute (chiasmal apoplexy) in $58 \%$ of patients, subacute in $15 \%$, and progressive in $26 \%$ [11]. In contrast, optic glioma typically manifests as slow, unilateral visual loss, proptosis, decreased color vision, optic-disc swelling or atrophy, or strabismus. Patients with more extensive tumors extending along the optic pathway may present with additional symptoms. Chiasmal gliomas usually present with slow bilateral visual loss associated with bitemporal field defects. Chiasmal apoplexy rarely occurs in an optic glioma, but the frequency is unknown. Malignant optic gliomas of adulthood usually present with sudden acute visual loss in 70$84 \%$ of patients [15]. The clinical symptoms and time courses of optic pathway CM and glioma often overlap. In our case, the time course of visual worsening could not differentiate between optic pathway CM and glioma.

\section{Neuroimaging findings of optic pathway CMs}

Optic pathway CM is characterized by heterogeneous appearance with mixed signal intensities suggestive of different stages of hemorrhage in $88 \%$ of cases, which is often described as "popcorn-like" lesion. T2-weighted imaging has shown a peripheral rim of hypointensity (hemosiderin ring) in $60 \%$ of cases. Minimal or no enhancement occurred after intravenous administration of gadolinium (Gd). Lesions of the optic nerve or tract tend to appear as a thickened nerve with increased diameter that is best seen on coronal images. The initial preoperative neuroimaging diagnosis was difficult to make, and $\mathrm{CM}$ was not suspected as the initial diagnosis in $34 \%$ of patients [11]. In contrast, the typical MR imaging findings of optic glioma include an iso- to hypointense lesion on T1-weighted images, a hyperintense lesion on T2weighted images, and homogeneous enhancement with $\mathrm{Gd}$ administration, although variable peripheral enhancement may be found with a central area of necrosis or cyst formation $[3,15]$. Chiasmal gliomas frequently demonstrate a variable contrast enhancement pattern and sometimes have suprasellar extension [5, 14]. If typical hemorrhagic findings are seen in the optic tract on MR imaging, CM should be considered in the differential diagnosis. However, if such typical neuroimaging findings are not obtained, $\mathrm{CM}$ is difficult to distinguish from optic glioma $[3,11,15]$.
Fig. 4 Fourth MR images, 2 weeks after the irradiation started and just before the operation, demonstrating further enlargement of the hemorrhage with increased heterogeneity (popcorn-like lesion)

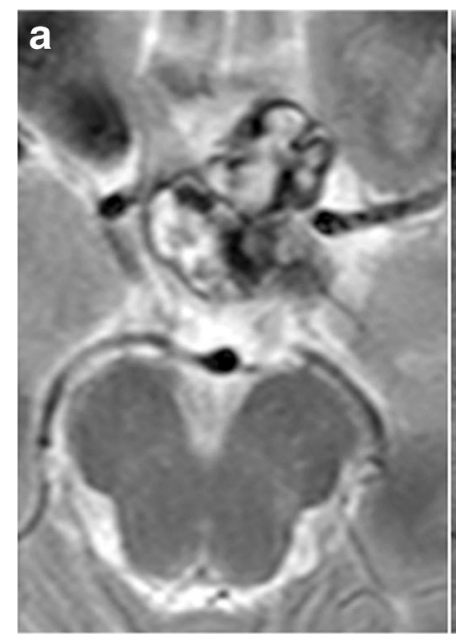

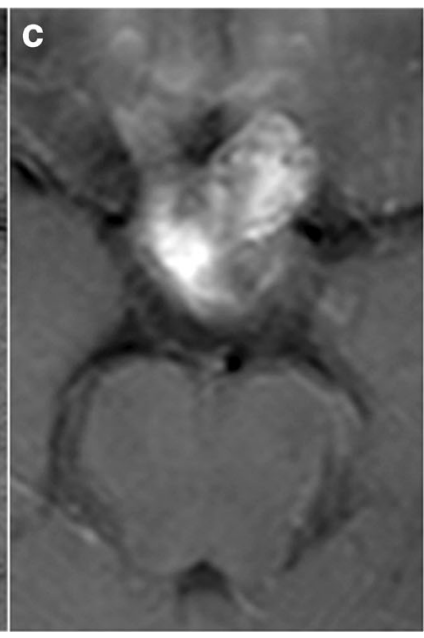


Fig. 5 Intraoperative views before (a) and after (b) removal of the lesion through the subfrontal approach with left frontal osteoplastic craniotomy. a Swelling of the left optic nerve with various stages of old hematoma. b The lesion extending from the left optic nerve to the chiasm and the left optic tract was totally resected. $L t$. IC left internal carotid artery
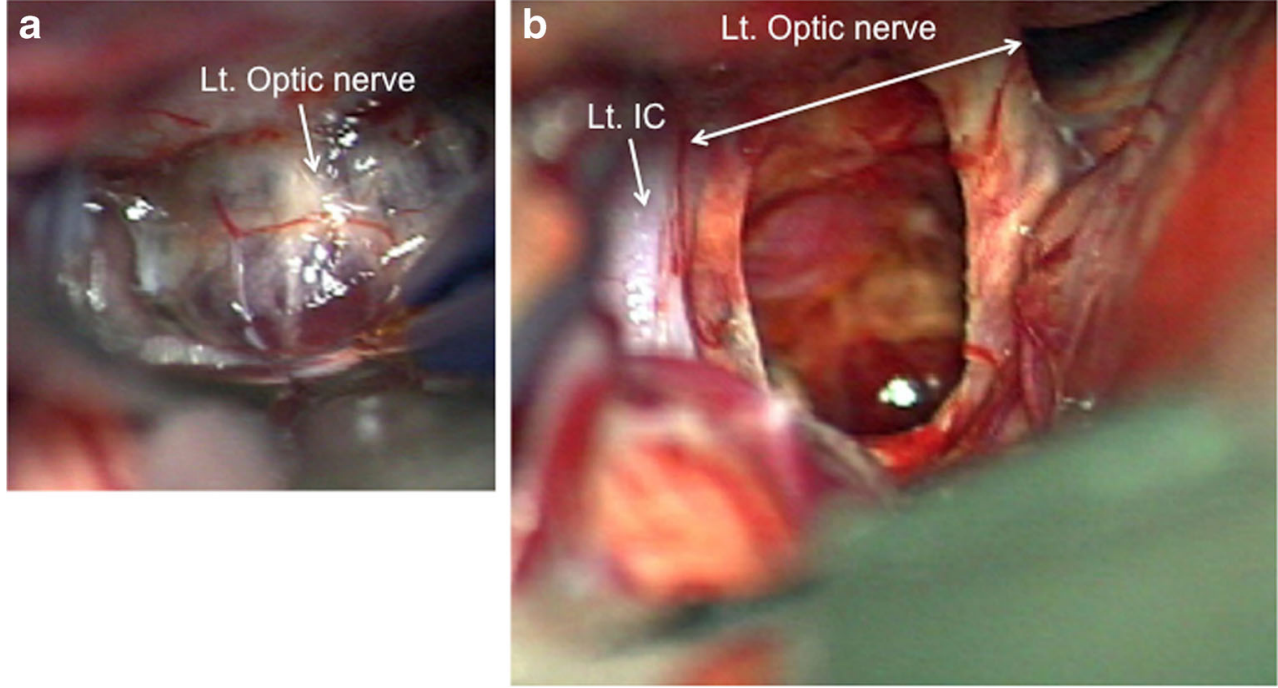

In our case, the initial MR images (Fig. 1a-d) did not show any typical hemorrhagic findings, such as popcorn-like lesion or hemosiderin ring. T1-weighted imaging with Gd showed peripheral ring enhancement. However, the initial T2weighted imaging (Fig. 1a) of the tumor showed low signal intensity. This finding was quite unusual as an optic glioma. The possibility of other diagnosis should be considered at that point, but the most likely diagnosis was optic glioma and optic $\mathrm{CM}$ did not pop into our mind at all. The second MR images (Fig. 2a-c) revealed hemorrhage associated with clinical aggravation. Most cases of optic glioma are pilocytic astrocytoma $[3,6]$. Hemorrhagic onset was observed in 4 of 35 patients with pilocytic astrocytoma and pilomyxoid astrocytoma. Hemorrhage is more common in pilocytic and pilomyxoid astrocytoma than in other types of neuroepithelial tumors [16]. Therefore, we considered that this hemorrhage was compatible with optic pilocytic/pilomyxoid astrocytoma. The possibility of optic pathway CM did not occur to us at all. The third (Fig. 3a-c) and fourth (Fig. 4a-c) MR images showed apparent enlargement of the hemorrhage associated with deterioration of her visual function. Retrospectively, the hemosiderin rim and popcorn-like lesion were apparent on the third MR images. Optic CM should have been suspected at this point. Therefore, the possibility of CM should be considered after detecting hemorrhagic findings in the optic region.

Optimal treatment of optic pathway CMs and management of optic pathway gliomas

Radical resection is the gold standard of treatment for optic pathway CMs, as any residual malformation carries the risk of rebleeding. Biopsy only is not recommended because of the risk of further bleeding and visual worsening [11]. Patients who were treated surgically and underwent gross total resection had the highest rate of visual improvement ( $85 \%$ ).

Careful observation may be acceptable in patients who are asymptomatic and have smaller optic pathway tumors [3]. Historically, there has not been clear consensus over the role of biopsy at diagnosis and the start of therapy against optic pathway tumors, independent of NF1 status. If an optic glioma

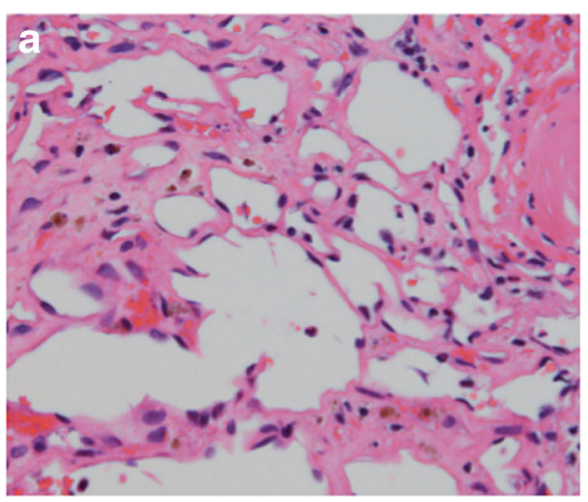

Fig. 6 Photomicrographs of the surgical specimen. a No malignant tissue pattern or atypical cells are observed. No neuroglial tissues are observed among the sinusoid chambers lined with flattened endothelia.

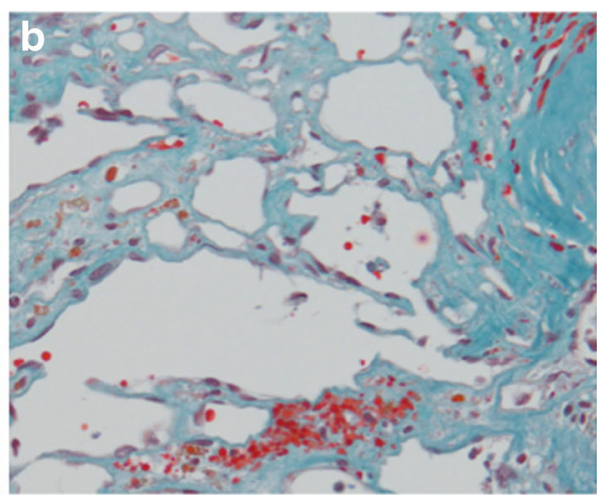

Hematoxylin and eosin staining, original magnification $\times 100$. b No component of elastic fiber or smooth muscle is present in the vessel walls. Elastica-Masson staining, original magnification $\times 100$ 
Fig. 7 Postoperative MR images (a-c). T1-weighted images with gadolinium demonstrated total removal of the optic $\mathrm{CM}$ and hematoma from the left optic nerve and chiasm
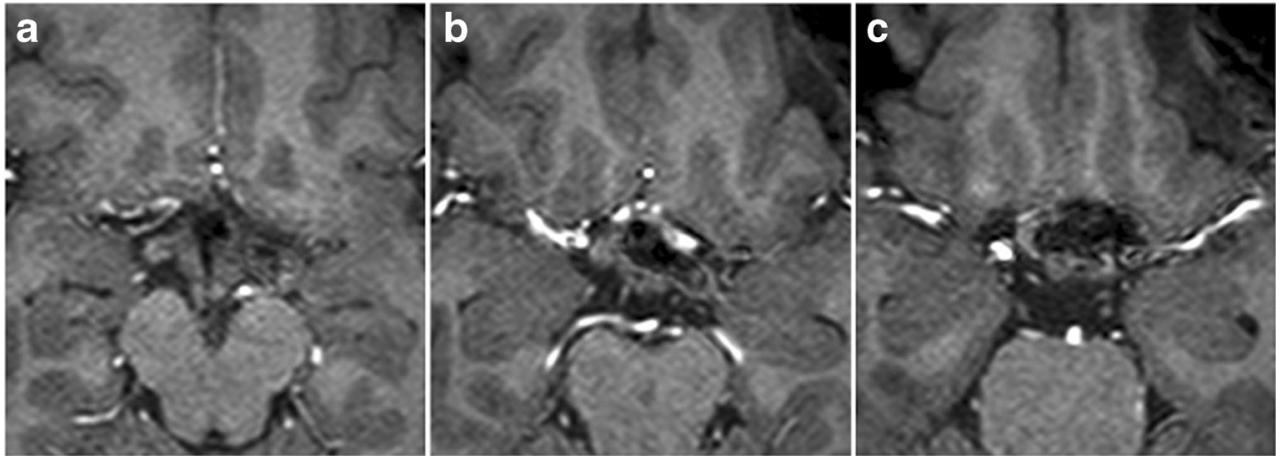

is highly suspected based on the MR imaging findings, that is included an iso- to hypointense lesion on T1-weighted images, a hyperintense lesion on T2-weighted images, and homogeneous enhancement or peripheral enhancement with Gd administration [3, 15], because most optic gliomas are low-grade astrocytoma, biopsy or other surgical interventions are not recommended $[18,20]$. As recent trends, optic pathway tumors with these typical MR imaging findings as optic gliomas do not require biopsy or surgery, and initially, watch/wait/rescan policy should be adopted if the tumor is small and asymptomatic [3]. Recent reported consensus statement concerning surgical approaches to pediatric brain tumors advocates that it is acceptable to perform a biopsy after multidisciplinary discussion by a specialist pediatric neurooncology team (including a pediatric neurosurgeon) before offering nonsurgical treatment (chemotherapy or biological therapy) in a clinical trial that involves a relevant biological stratification or question for NF1-negative/sporadic optic gliomas [19].

Patients presenting with symptoms of visual loss, endocrine disturbance, hydrocephalus, or mass effect may require aggressive intervention. They reported some significances of biopsy and debulking surgery; those can determine histopathology and relief of mass effect $[4,7]$. Although, recent indications for surgery are limited to single nerve involvement causing progressive disfiguring proptosis, blindness, or both, or exophytic chiasm tumors causing mass effect or hydrocephalus [3]. The consensus allows to perform surgical debulking for patients with some characteristics (e.g., cystic tumors, large tumors, and exophytic or hypothalamic tumors) when early surgical debulking is possible with acceptable risk based on multidisciplinary discussion [19].

Given the risks of surgery and radiotherapy, chemotherapy is the initial treatment for most optic gliomas [1]. Recently, platinum-based chemotherapy has been preferred as the firstline treatment for all ages, and continuous irradiation might be recommended if the patient is older than 5 years $[9,10]$. When intervention would be needed, it was acceptable to start platinum-based chemotherapy without histological diagnosis for patients with typical MR findings. However, it may be necessary to perform biopsy to the optic lesion with unusual MR findings like our case.
The optimal treatment of optic pathway CMs is radical resection. In contrast, surgical resection of optic glioma cannot be recommended except in particular situations. Our patient underwent unnecessary radiochemotherapy because the unusual neuroimaging findings were suggestive of optic glioma rather than optic pathway CM. Hemorrhage was identified on the second and third MR images. Such findings should indicate immediate radical resection without radiochemotherapy.

\section{Conclusions}

The present unusual case of optic pathway CM mimicking optic glioma shows that even if optic glioma is suspected based on the neuroimaging findings, optic pathway CM should be considered in the differential diagnoses. If findings of hemorrhage are obtained, immediate radical resection should be performed to preserve residual visual functions and to establish the definitive diagnosis.

Conflict of interest The authors declare that they have no conflict of interest.

\section{References}

1. Avery RA, Fisher MJ, Liu GT (2011) Optic pathway gliomas. J Neuroophtalmol 31:269-278

2. Batra S, Lin D, Recinos PF, Zhang J, Rigamonti D (2009) Cavernous malformations: natural history, diagnosis and treatment. Nat Rev Neurol 5:659-670

3. Binning MJ, Liu JK, Kestle JR, Brockmeyer DL, Walker ML (2007) Optic pathway gliomas: a review. Neurosurg Focus 23(5):E2

4. Bommakanti K, Panigrahi M, Yarlagadda R, Sundaram C, Upin MS, Purohit AK (2010) Optic chiasmatic-hypothalamic gliomas: is tissue diagnosis essential? Neurol India 58:833-840

5. Cummings TJ, Provenzale JM, Hunter SB, Friedman AH, Klintworth GK, Bigner SH, McLendon RE (2000) Gliomas of the optic nerve: histological, immunohistochemical (MIB-1 and p53), and MRI analysis. Acta Neuropathol 99:563-570

6. Dutton JJ (1994) Gliomas of the anterior visual pathway. Surv Ophthalmol 38:427-452 
7. Goodden J, Pizer B, Pettorini B, Williams D, Blair J, Didi M, Thorp N, Mallucci C (2014) The role of surgery in optic pathway/hypothalamic gliomas in children. J Neurosurg Pediatr $12: 1-12$

8. Gross BA, Lin N, Du R, Day AL (2011) The natural history of intracranial cavernous malformations. Neurosurg Focus 30(6):E24

9. Jahraus CD, Tarbell NJ (2006) Optic pathway gliomas. Pediatr Blood Cancer 46:586-596

10. Liu GT (2006) Optic gliomas of the anterior visual pathway. Curr Opin Ophthalmol 17:427-431

11. Liu JK, Lu Y, Raslan AM, Gultekin SH, Delashaw JB Jr (2010) Cavernous malformations of the optic pathway and hypothalamus: analysis of 65 cases in the literature. Neurosurg Focus 29(3):E17

12. Murai Y, Adachi K, Koketsu K, Teramoto A (2011) Indocyanine green videoangiography of optic cavernous angioma - case report. Neurol Med Chir (Tokyo) 51:296-298

13. Ning X, Xu K, Luo Q, Qu L, Yu J (2012) Uncommon cavernous malformation of the optic chiasm: a case report. Eur J Med Res 17:24

14. Pepin SM, Lessell S (2006) Anterior visual pathway gliomas: the last 30 years. Semin Ophthalmol 21:117-124
15. Shapey J, Danesh-Meyer HV, Kaye AH (2011) Diagnosis and management of optic nerve glioma. J Clin Neurosci 18:1585-1591

16. Shibahara I, Kanamori M, Kumabe T, Endo H, Sonoda Y, Ogawa Y, Watanabe M, Tominaga T (2009) Hemorrhagic onset of pilocytic astrocytoma and pilomyxoid astrocytoma. Brain Tumor Pathol 26:1-5

17. Uppal S, Walker RA, Atkins EJ (2012) Cavernous malformation of the optic chiasm - a diagnostic and treatment dilemma. Can J Neurol Sci 39:533-535

18. Varan A, Batu S, Cila A, Soylemezoglu F, Balci S, Akalan N, Zorlu F, Akyuz C, Kutluk T, Buyukpamukcu M (2013) Optic glioma in children: a retrospective analysis of 101 cases. Am J Clin Oncol 36: 287-292

19. Walker DA, Liu J, Kieran M, Jabado N, Picton S, Packer R, St Rose C, CPN Paris (2011) Conference Consensus Group (2013) A multidisciplinary consensus statement concerning surgical approaches to low-grade, high-grade astrocytomas and diffuse intrinsic pontine gliomas in childhood (CPN Paris 2011) using the Delphi method. Neuro Oncol 15:462-468

20. Wilhelm H (2009) Primary optic nerve tumours. Curr Opin Neurol 22:11-18 